\title{
USO INDISCRIMINADO E/OU IRRACIONAL DE ANTIINFLAMATÓRIOS NÃO ESTEROIDAIS (AINES) OBSERVADOS EM UMA FARMÁCIA DE DISPENSAÇÃO
}

\author{
INDISCRIMINATE USE AND/OR THE IRRATIONAL NON-STEROIDAL ANTI- \\ INFLAMATORY (NSAID) OBSERVED IN A PHARMACY OF DISPENSING
}

\author{
Fabiane VILETTI'; Andréia Cristina Conegero SANCHES ${ }^{2}$ \\ ${ }^{1}$ Fabiane Viletti, Curso de Especialização em Farmacologia e Farmácia de \\ Dispensação, UNIVERSIDADE ESTADUAL DO OESTE DO PARANÁ \\ ${ }^{2}$ Andréia Cristina Conegero Sanches, docente e coordenadora do Curso de Especialização em Farmacologia e \\ Farmácia de Dispensação - UNIVERSIDADE ESTADUAL DO OESTE DO PARANÁ - Campus Cascavel/PR, \\ andréia_cvel@hotmail.com \\ REC:002/09 AC:03/09
}

\begin{abstract}
RESUMO:
Os antinflamatórios não-esteroidais são utilizados para dores pós-operatórias, artrite reumatóide, músculos esqueléticos e osteoartrites, seus principais efeitos são antiinflamatório, analgésico e antipirético. Alem da atividade terapêutica esses fármacos compartilham efeitos indesejáveis, os mais evidentes são complicações gastrintestinais. Objetivos: observar, analisar e avaliar a utilização de medicamentos antiinflamatórios, Metodologia: Estudo do tipo observacional, onde a coleta de dados em farmácia de dispensação, por meio de entrevista estruturada a pessoas que buscavam antiinflamatório não-esteroidal com ou sem prescrição médica, de acordo com os preceitos éticos de pesquisa. Foram avaliadas as variáveis sociodemográficas e farmacoepidemiologicas, por análise estatística descritiva. Resultados e conclusão: Os principais fatores associados aos AINEs foram o sexo feminino e a jornada de trabalho longa e cansativa, sendo a dor o fator predominante. A freqüência diária de uso entre $60 \%$ dos entrevistados nos leva a concluir que a falta de informação faz com que as pessoas utilizem antiinflamatórios nãoesteroidais de forma indiscriminada, sem perceber que outros problemas de saúde podem estar sendo desenvolvidos ou agravados por esta conduta, a importância do profissional da saúde na intervenção desse ato é de suma importância.
\end{abstract}

Palavras - chave: Antiinflamatórios não esteroidais (AINEs), dor, uso indiscriminado.

\begin{abstract}
:
Non-steroidal anti-inflammatory are used for postoperative pain, rheumatoid arthritis, and osteoarthritis skeletal muscles, its main effects are anti-inflammatory, analgesic and antipyretic. Besides the therapeutic activity such drugs share non-pleasant effects, the most obvious are gastrointestinal complications. Objective: To observe, analyze and evaluate the use of antiinflammatory drugs. Methodology: Data collection from dispensing pharmacy, where people who sought non-steroidal anti-inflammatory with or without prescription were interviewed. We evaluated the social demographic and pharmacoepidemiology variables, by descriptive statistical analysis. Results and conclusion: The main factors associated with NSAIDs have been among the female sex and the long and tiring working hours, pain being the predominant cause. The frequency of daily use among $60 \%$ of respondents draw us to conclude that the lack of information leads people to use non-steroidal anti-inflammatory drugs in an indiscriminately way, without realizing that other health problems are being developed or aggravated by this behavior, the relevance of a health professional intervention in this matter is of paramount importance.
\end{abstract}

Key-words: Non-steroidal Anti-inflammatory agents (NSAIDs), pain, indiscriminate use. 


\section{INTRODUÇÃO}

Os antiinflamatórios não esteroidais (AINEs) são utilizados no tratamento de dor pós-operatória, osteoartrite, artrite reumatóide e dores músculo-esqueléticas, entre diversas condições. Seus principais efeitos são antiinflamatório, analgésico e antipirético (ARAUJO, et al., 2005). Em geral, esses efeitos estão relacionados à inibição da enzima cicloxigenase, que catalisa a transformação de acido araquidônico em diversos mediadores lipídicos, denominados prostaglandinas e tromboxanos, estas substâncias tem importante função homeostática na proteção da mucosa gástrica, fisiologia renal, gestação e agregação plaquetária, além de terem sua produção induzida em condições como inflamação e câncer, estudos envolvendo AINEs com seletividade para cicloxigenase-2 evidenciaram maior risco de eventos adversos cardiovasculares como infarto agudo do miocárdio, acidente vascular cerebral, hipertenção e insuficiencia cardíaca (ARAUJO et al., 2005).

Existem duas isoformas mais conhecidas da enzima cicloxigenase, que são a cicloxigenase1 (COX -1) e a cicloxigenase 2 (COX-2). Recentemente foi descoberta uma variante do gene da COX-1, descrito como COX-3 que parece ser expressa em altos níveis no sistema nervoso central e pode ser encontrada também no coração e na aorta. Esta enzima é seletivamente inibida por drogas analgésicas e antipiréticas, e é pontencialmente inibida por alguns AINEs, tal inibição pode representar um mecanismo primário central pelo qual essas drogas diminuem a dor e possivelmente a febre. A relevância dessa isoforma ainda não está clara (MONTEIRO et al., 2008). A enzima cicloxigenase 1 é uma enzima constitutiva expressa na maioria dos tecidos, incluindo as plaquetas sanguíneas, e está envolvida na sinalização entre células e na homeostasia tecidual. A cicloxigenase 2 é induzida nas células inflamatórias quando estas são ativadas, e, neste aspecto, as citocinas inflamatórias primarias - interleucina 1 e fator de necrose alfa - são importantes (RANG et al.,2001).

Os salicilatos e os antiinflamatórios não esteroidais mais antigos inibem tanto a COX-1 quanto a COX-2, com baixa seletividade sobre a COX-2, diferindo dos AINEs inibidores seletivos da COX-2 desenvolvidos mais recentemente. As ações antiinflamatórias, analgésica e antipirética dos AINEs decorrem principalmente da ação inibitória sobre a COX-2, enquanto os efeitos colaterais são resultantes notadamente da inibição da COX-1 (SILVA, 2006).

Alem de compartilhar muitas atividades terapêuticas, essas drogas também compartilham vários efeitos colaterais indesejáveis. O mais comum consiste na tendência a induzir ulceração gástrica ou intestinal que, algumas vezes, pode ser acompanhada de anemia devido a conseqüente perda de sangue. Uma notável exceção é representada pelos inibidores altamente seletivos da COX-2 que não tem propensão a causar ulceração gástrica. Os pacientes que usam essas drogas não seletivas, de forma crônica correm um risco relativo cerca de três vezes maior de sofrer eventos gastrintestinais adversos graves em comparação com não usuários (GOODMAN \& GILMAN, 2003).

O provável mecanismo para tal toxicidade é a inibição da atividade da isoforma COX-1, que produz as prostaglandinas citoprotetoras $\mathrm{PGE}_{2}$ e $\mathrm{PGI}_{2}$. Estas são responsáveis pela manutenção da integridade da mucosa gástrica, reduzem a secreção 
de ácido, aumentam a secreção de bicarbonato e melhoram o fluxo sangüíneo na microcirculação da mucosa. O sangramento gastrointestinal relaciona-se também à inibição da atividade da COX-1 plaquetária (KUMMER et al., 2002).

Outra ação tóxica é a hepatotoxicidade, o espectro clínico dessa lesão varia desde elevações assintomáticas das aminotransferases até quadros de hepatite aguda grave com icterícia hepatocelular, evoluindo para óbito. A população particularmente vulnerável a essa complicação engloba pacientes do sexo feminino, idosos, portadoras de osteoartrites (GRAF et al., 2002).

Os antiinflamatórios não-esteróides integram o grupo dos fármacos mais comumente prescritos em todo o mundo e estão entre os mais utilizados nas práticas de automedicação. Nos Estados Unidos, respondem por mais de 70 milhões de prescrições e mais de 30 bilhões de comprimidos de venda livre comercializados anualmente. No Brasil, diferentes estudos de utilização de medicamentos situam os AINE entre os mais utilizados pela população (RIBEIRO et al., 2005).

Como esta classe de medicamentos é largamente utilizada no mundo, vários estudos foram realizados sobre a eficácia, tolerância e agravantes que poderiam ocorrer com a utilização dos mesmos.

A utilização de antiinflamatórios não esteroidais, os chamados AINEs, vêm crescendo amplamente. Assim, faz-se necessário, estudos que mostrem a racionalidade da população sobre o uso desses medicamentos, assim este estudo busca avaliar a utilização de antiinflamatórios não esteroidais prescritos e não prescritos em uma farmácia de dispensação.

\section{METODOLOGIA}

O trabalho foi realizado numa farmácia de dispensação, localizada em um bairro do município de Toledo - PR. Foram entrevistadas pessoas com faixa etária acima de 18 anos, que buscam antiinflamatório não esteroidal com ou sem prescrição médica e de qualquer forma farmacêutica, elas foram orientadas sobre os preceitos éticos na pesquisa e autorizaram a coleta de dados. Foram avaliadas as variáveis sociodemográficas e farmacoepidemiologicas, num segundo momento os pacientes foram informados sobre o uso racional desses medicamentos. Os dados foram avaliados através de análise estatística descritiva.

\section{RESULTADOS E DISCUSSÃO}

Foram entrevistadas aleatoriamente 30 pessoas que buscaram a farmácia no período de fevereiro a maio de 2008 e buscaram antiinflamatórios não-esteroidais com e sem prescrição de profissional habilitado (médico e/ou dentista).

Diante de tal levantamento observou-se que $60 \%$ das pessoas entrevistadas eram do sexo feminino e $40 \%$ do sexo masculino. Segundo Garcia J.B.S, (2007) o sexo feminino sente mais dor e está mais susceptível a essa sensação, por isso são mais comuns em mulheres dores nos ombros, no abdômen, dores de cabeça entre outras. Esse trabalho está de acordo com os estudos existentes que mostram a maior procura por drogas que reduzem as dores pelo sexo feminino. Além disso, observou- 
se o nível de escolaridade dos entrevistados onde 16,7\% nível superior; $13,3 \%$ ensino médio completo, $10 \%$ não concluíram o ensino médio; $46,76 \%$ ensino fundamental incompleto, 3,3\% ensino fundamental completo e 10\% são analfabetos. Luz et al(2003) declarou em seu trabalho sobre o uso de AINEs em uma população de funcionários de uma universidade no Rio de Janeiro, que fatores como o nível de escolaridade, econômico e social são muito difíceis de serem interpretados como influentes em um estudo envolvendo uso de medicamentos, já que trabalhos realizados em outros países entram em controvérsias sobre esse tipo de relevância.

Entre o grupo avaliado observou-se que quem mais faz uso de AINES está entre 41 e 50 anos de idade com 33,3\% dos usuários e a que menos faz uso é a faixa etária de 21 à 30 anos com 6,6\%. Rozenfeld, (2003), diz que entre os fatores preditores do uso irracional de medicamentos estão a idade avançada, o sexo feminino, as piores condições de saúde e a depressão, onde as classes terapêuticas mais consumidas são as cardiovasculares, os anti-reumáticos e os analgésicos.

Os motivos que levam as pessoas usarem esses medicamentos são dores relacionadas diretamente à jornada de trabalho, na maioria das vezes trabalho braçal e jornadas longas, sendo eles, em grande numero, trabalhadores rurais e secretárias do lar, onde se enquadra a faixa etária de 41 à 60 anos somando 56\% dos entrevistados. Afirmando esta estatística, Luz et al (2003), diz em seus estudos que mulheres e indivíduos com maior carga horária de trabalho semanal constituem grupos mais vulneráveis, em termos de uso irracional.

Quando lhes foi questionado quanto à finalidade de uso de tal droga obteve-se como resposta os mais diversos tipos de dores, entretanto os mais relatados foram dores musculares, lombares, articulações, ou seja, dores relacionadas a algum tipo de esforço que são expostos com freqüência. Outros tipos de dores também foram evidenciados, como garganta, para tal observou-se que a faixa etária que mais faz uso para esta finalidade é de 21 a 40 anos com nível de escolaridade maior. Dores renais, pós cirúrgicas, cólicas, ácido úrico, hemorróidas também foram citadas com menor freqüência de uso de AINES. Segundo Andrade, et al(2006) a dor está entre os principais fatores que podem impactar negativamente a qualidade de vida do paciente com mais idade, pois limita suas atividades, aumenta a agitação, o risco para estresse e o isolamento social.

Outro dado foi quanto a relação uso prescrito/automedicação. Entre os entrevistados $36,7 \%$ vieram com prescrição em mãos, $46,7 \%$ estavam fazendo uso do medicamento por indicação de amigos, parentes e/ou vizinhos e 16,76\% haviam sido orientados por profissional habilitado (médico ou dentista) a fazer uso, mas uma única vez e a partir daí fazem uso freqüentemente, pois alegam que se voltarem ao médico tal antiinflamatório será prescrito novamente, então preferem ir diretamente à farmácia. Vitor et al. (2008) destaca que a automedicação é praticada universalmente e está presente nas mais diferentes sociedades e culturas inclusive por mais da metade da população brasileira. Loyola Filho et al. (2004), verificou que a influencia de pessoas próximas, a percepção de pouca gravidade do problema, a familiaridade e o fácil acesso a alguns medicamentos, bem como a disponibilidade e a percepção do atendimento médico recebido como de pouca qualidade, foram fatores determinantes da automedicação. 
Quanto à eficácia do medicamento para o problema que buscavam solução, $100 \%$ das pessoas responderam positivamente. Os AINES tem ótima atividade analgésica e antiinflamatória, por isso é uma das classes de medicamentos mais dispensadas nas drogarias. Em um estudo envolvendo um AINE especifico, sendo ele o diclofenaco colestiramina que foi utilizado em síndromes dolorosas lombares, Sakurada (2001) concluiu que o tratamento, nas condições do trabalho, foi eficaz e bem tolerado. Entretanto estudos são necessários para avaliar os riscos do uso indiscriminado de antiinflamatórios por longo período, tendo em vista todos os riscos já descritos desses medicamentos.

Quanto à forma farmacêutica, a mais dispensada foi comprimido, com $56 \%$ da preferência, e em seguida o gel com $26 \%$, os $18 \%$ restantes foram a dispensação desta classe na forma farmacêutica de supositório, gotas, aerossol e injetável. A praticidade do comprimido pode ser um fator que explique esta preferência. Percebeuse que procura de supositórios, injetáveis, granulados e gotas na maioria das vezes foi mediada por prescrição de profissional habilitado.

A freqüência de uso foi um fator bem significativo, pelo menos $60 \%$ dos entrevistados tomam antiinflamatórios não esteroidas todos os dias. Além disso, $54 \%$ das pessoas citaram algum tipo de comprometimento do tracto gastro intestinal entre outros problemas de saúde como hipertensão, diabetes, problemas hepáticos e renais. Todos AINEs convencionais têm a tendência de causar efeitos adversos gastrointestinais que podem variar de dispepsia a sangramentos de estômago e duodeno, ativar doenças inflamatórias intestinais quiescentes e causar dano tecidual (como úlceras) no trato gastrointestinal baixo, geralmente, após um longo período de uso (MONTEIRO et al., 2008).

Segundo RIBEIRO et al., (2005), o uso destes fármacos e a presença de sintomas gastrintestinais é um fato preocupante que merece atenção especial na prática médica e deve ser colocado como objeto de futuras investigações farmacoepidemiológicas. TOGNINI et al.,(1998) em um estudo realizado com ratos concluiu que a cicatriz da parede abdominal de rato tratado com o diclofenaco de sódio apresenta menor quantidade de fibras colágenas, quando comparadas ao grupo controle. Na verdade muitas pessoas fazem uso de medicamentos sem saberem que podem agravar problemas de saúde já existentes, ou ainda provocarem um novo problema como lesões gastrintestinais que são comprovadamente causadas com o uso de antinflamatório não esteroidais convencionais. Kummer (2002) cita que o uso de AINES convencionais está relacionado a alto risco de dano na mucosa gastrointestinal, incluindo úlceras, erosões e, ainda, complicações como sangramento gastrointestinal, perfuração e obstrução. Diz ainda que pacientes com úlcera gástrica diagnosticada devem preferentemente ter outra forma de analgesia que não seja AINES enquanto se submetem ao tratamento da lesão. GRAF et al.,(2002) verificou em seu trabalho que antiinflamatórios não hormonais podem ser causa de lesão hepática, sendo esta determinada muito mais por mecanismo idiossincrático do que por efeito tóxico direto, descreve-se um caso de hepatotoxicidade grave por diclofenaco, um dos antiinflamátorios mais utilizados em nosso meio. KUMMER (2002) e BANKS et al.(2001) também afirmam que antiinflamatórios não-esteroidais, particularmente diclofenaco de sódio, têm sido associados a graves quadros de hepatotoxicidade. Hepatite tem 
sido relacionada ao uso do diclofenaco (MONTEIRO et al., 2008).

Quando foram questionados sobre outros medicamentos que faziam uso sem prescrição médica, o diclofenaco foi mencionado prontamente, onde relataram fazer uso quando sentiam muita dor. Ao questioná-los sobre os medicamentos que fazem parte de sua "sacola de medicamentos domiciliar" obeservou-se que estavam adquirindo um fármaco com o mesmo efeito terapêutico daquele que já possuía em sua casa. Dentre os medicamentos mais usados sem a devida prescrição, a amoxilina, os relaxantes musculares e o omeprazol foram os mais citados. Quando questionou-se sobre medicamentos que tinham em casa mas desta vez prescritos por profissionais habilitados os anti-hipertensivos ganharam maior destaque. KUMMER (2002) relatou que lesões agudas gastrointestinais estão entre os efeitos colaterais mais frequentes e graves associados com AINES convencionais e que o risco de perfuração e ulceração gástrica apresenta incidência três a quatro vezes maiores em usuários destes compostos.

Outro inconveniente é a hipertensão arterial, pois a exacerbação da hipertensão com o uso de AINEs continua a ser um ponto controverso entre os pesquisadores. Estes medicamentos segundo Plavnik, F. L. da Revista Bras. Hipertens vol 9 de 2002 podem induzir aumento na pressão arterial ou mesmo interferir na eficácia dos antihipertensivos.

Através dos dados obtidos, analisados e discutidos neste trabalho, fica claro a falta de informação e até mesmo a falta de um acompanhamento profissional para orientar e conscientizar as pessoas de que medicamento deve ser utilizado com cautela, pois as conseqüências podem ser serias. Um trabalho muito importante é o de atenção farmacêutica que tem por objetivo maior, a utilização adequada e racional dos fármacos garantindo assim a qualidade de vida do paciente. O profissional da saúde deve estar atento sempre, e, ser a peça fundamental para levar a informação às pessoas que necessitam, intervindo quando for necessário e ajudando a solucionar os problemas de saúde que atribulam o seu dia a dia.

\section{CONCLUSÃO}

O uso indiscriminado de medicamentos de uma maneira geral pode ser considerado preocupante, e, com os AINES esta preocupação não é diferente já que entre os entrevistados muitos, utilizam esses fármacos para as diversas peculiaridades da rotina diária (como dores provenientes do esforço físico). A dor é um determinante para a grande procura de antiinflamatórios, pois se mostram eficazes para o alivio da mesma. Mais procurados por mulheres e pessoas expostas ao trabalho pesado com carga horária extensa, munidos ou não de prescrição médica e/ou odontológica, esses medicamentos são uma das classes mais utilizadas e são potenciais agravantes e/ou causadores de problemas gástricos e intestinais, como ulcerações.

Por meio dos dados obtidos, torna-se claro a falta de informação e até mesmo a falta de um acompanhamento profissional para orientar e conscientizar as pessoas de que medicamento deve ser utilizado com cautela, pois as conseqüências podem ser sérias. O profissional da saúde deve estar atento sempre, e, ser a peça fundamental para levar a informação às pessoas que necessitam. 


\section{REFERÊNCIAS}

ANDRADE, F.A; PEREIRA, L.V.; SOUSA, F.A.E.F. - Mensuração da dor no idoso: uma revisão. Revista Latino-Americana Enfermagem; 14(2): 271-6, 2006.

ROZENFELD, S. Prevalência, Fatores Associados e mal uso de medicamentos entre idosos: uma revisão. Cad. Saúde Publica, 19 (3): 717-724, 2003.

ARAÚJO, L. F.; SOEIRO, A. M.; FERNANDES, J.L.; SERRANO JUNIOR, C. V. Eventos Cardiovasculares: Um Efeito de Classe dos Inibidores de COX-2. Arquivos Brasileiros de Cardiologia, v. 85, no 3, 2005.

BANKS, A. T; ZIMMERMAN, H. J; ISHAK, K. G; HARTER, J.G. Hepatotoxicidade associada ao diclofenaco: análise de 180 casos relatados a Food and Drug Administration como reaçöes adversas. Folha Medica 120(1):52-60, jan.-mar. 2001.

LUZ, T. C. B. - Fatores associados ao uso de antiinflamatórios não esteroidais em população de funcionários de uma universidade do Rio de Janeiro. Revista Brasileira de Epidemiologia; 9 (3); 2003.

GARCIA, J. B. S. Ano Internacional de Combate a Dor na mulher. Jornal Dor Ano VII - 4ํㅡㅁ Trimestre de 2007. Disponível em: http://dor.org.br/images/jornal26.pdf. Acesso em 04/08/2008.

GRAF, J.; RUMOR, C.; FONSECA, V. R. C. D. Hepatite causada pelo uso de diclofenaco sódico. Revista Gastroenterol. Endosc. Dig; 21(2):82-84, 2002.

GOODMAN \& GILMAN. As Bases Farmacológicas da Terapêutica. AnalgésicoAntipiréticos, Agentes Antiinflamatórios e Fármacos Utilizados no Tratamento da Gota. 10ํㄹição. Rio de Janeiro: Guanabara Koogan, 2003.

KUMMER, C. L.; COELHO, T. C. R. B. Cycloxygenase-2 inhibitors nonsteroid antiinflammatory drugs: current issues. Revista Brasileira de Anestesiologia, vol.52,n.4, 2002.

LOYOLA FILHO, A. I. ; LIMA-COSTA, M. F.; UCHOA, E. Bambuí Project: a qualitative approach to self-medication. Cad. Saúde Pública, Rio de Janeiro, v. 20, n. 6, 2004.

MONTEIRO, E. C. A.; TRINDADE, J. M. F.; DUARTE, Â. L. B. P.; CHAHADE, W. H. Os Antinflamatórios não Esteroidais ( AINEs) Revista Brasileira de Medicina Disponível em http://www.cibersaude.com.br/ revistas.asp?fase=r003\&id_materia=3744, acesso em 20/09/2008.

PLAVNIK, Frida Liane - Hipertensao Arterial induzida por Drogas: Como Detectar e 
Tratar - Revista Bras. Hipertens., vol 9:2; abril/junho de 2002.

RANG, H.P.; DALE, M.M.; RITTER. J M. Farmacologia. Agentes Antiinflamatórios e Imunossupressores. 4ำ edição. Rio de Janeiro: Guanabara Koogan, 2001.

RIBEIRO, Andréia Queiroz; SEVALHO, Gil; CESAR, Cibele Comini - Prevalência e fatores associados ao uso de antiinflamatórios não-esteróides por pacientes submetidos a endoscopia digestiva alta/ Prevalence of and factors associated with nonsteroidal anti-inflammatory drug use by patients submitted to upper endoscopy Revista Brasileira de Epidemiologia, 8(3):306-315, set. 2005.

SAKURADA, Cláudio; PASOTO, Sandra Gofinet; KANEGAE, Márcia - Avaliaçäo em larga escala do diclofenaco colestiramina em síndromes dolorosas lombares, Rev.

Bras. Med; 58 (11):850-858, nov. 2001.

SILVA, Penildon. Farmacologia. Atiinflamatórios Não- Esteróides, Analgésicos, Antipiréticos e Drogas Utilizadas no Tratamento da Gota. $7^{\circ}$ Edição. Rio de Janeiro: Guanabara Koogan, 2006.

TOGNINI, João Ricardo F.; GOLDENBERG, Saul; SIMÕES, Manuel de Jesus; SAUER, Leandro; MELO, Rosana Leite de; ORTIZ, Pedro Leopoldo A. Efeito do Diclofenaco de Sódio na Cicatrização da Parede Abdominal de Ratos. Acta Cirurgica Brasileira, vol.13, n. 3, 1998.

VITOR, Ricardo Sozo et al. Padrão de consumo de medicamentos sem prescrição médica na cidade de Porto Alegre, RS. Ciênc. Saúde Coletiva, vol.13, suppl., pp. 737-743, 2008. 